\title{
Carlos Maya-Ambía
}

\section{Globalisierung der Ökonomie, Polarisierung der Macht Dilemmata der US-amerikanischen Hegemonie}

Zum ersten Mal in der Geschichte ist man in Washington der Ansicht, die ganze Welt sei der Hinterhof der USA. Der zweite Irak-Krieg zeigte, dass George W. Bush in einer Welt der Kreuzzüge lebt, in der die Mission der USA darin besteht, „das Böse“ zu bekämpfen. Um dieses Ziel zu verfolgen, hat sich Washington entschieden, die Welt - zumindest die Dritte Welt - in eine ihrer Einflusssphären zu transformieren. Angesichts der gegenwärtigen Unipolarität der Weltpolitik ist die "Lateinamerikanisierung" der Welt zu Beginn des 21. Jahrhunderts eine ernste Gefahr für die Menschheit.

Die gegenwärtige - unipolare - Situation, die im ersten Teil skizziert wird, sollte im Kontext der Entwicklung der US-amerikanischen Hegemonie betrachtet werden. Auf den folgenden Seiten ist es daher mein Anliegen, einige Überlegungen zum Rekonstruktionsprozess der US-amerikanischen Hegemonie zur Diskussion zu stellen. Da ich dabei den neogramscianischen Ansatz zur Analyse der Internationalen Beziehungen zugrunde lege, werde ich dieses Konzept kurz erläutern. Anschließend versuche ich, auf dieser Basis die Krise und die Wiederherstellung der US-amerikanischen Hegemonie zu analysieren.

\section{Unipolarität und die "Bush-Doktrin" ${ }^{1}$}

Die Hegemonie der Vereinigten Staaten basiert auf zwei zentralen Strategien, die sich für die Gestalt der internationalen Ordnung als entscheidend erwiesen haben. Die eine könnte man die „realistisch ausgerichtete“ Strategie nennen, da sie auf die Aufrechterhaltung der globalen Machtbalance durch Gefahrenabwehr und Abschreckung abzielt. Solange es um den Erhalt des bipolaren Gleichgewichtes zwischen den USA und der früheren Sowjetunion ging, hat sich diese Strategie bewährt (Ikenberry 2002: 45).

1 Um dem Verdacht „antiamerikanischer" Vorurteile in meiner Argumentation vorzubeugen, beziche ich mich in diesem Aufsatz und insbesondere in diesem Teil auf verschiedene nichtmarxistische Autoren. Die meisten von ihnen sind amerikanische Wissenschaftler, die der US-Außenpolitik kritisch gegenüberstehen. 
Die zweite, „liberale" Strategie versucht die Öffnung der Ökonomien voranzutreiben, ün nicht in die Situation der 30er Jahre zurückzufallen, in die Zeit der regionalen Blöcke, Handelskonflikte und strategischen Rivalitäten. Beide Strategien funktionierten während des Kalten Krieges, aus dem die USA als Sieger hervorgingen. Ihr Triumph gipfelte schließlich in einer unipolaren Welt. Huntington zufolge ist "Unipolarität" ein System mit einer Supermacht und vielen kleinen Mächten aber ohne wesentliche Großmächte. Die innerhalb eines solchen Systems dominierende Macht sei fähig, „relevante internationale Probleme effektiv allein zu lösen, wobei kein Zusammenschluss anderer Staaten mächtig genug ist, sie davon abzuhalten" (Brooks/Wohlfort 2002: 21). Folgt man dieser Idee, dann umfasst die gegenwärtige Unipolarität drei Bereiche: Militär, Ökonomie und Technologie. Bemerkenswert am bestehenden internationalen System ist die US-amerikanische Vorherrschaft in jedem dieser drei Felder (ebd.: 23). Diese Situation hat die Herausbildung einer neuen Strategie ermöglicht. Sie gründet sich auf die Tatsache, dass die Vereinigten Staaten im Umgang mit terroristischen Bedrohungen zunehmend unilateral und präventiv und mutmaßliche "Schurkenstaaten" des Besitzes von Massenvernichtungswaffen beschuldigen. Dabei nehmen sie weder auf ihre Partner noch auf die globalen Regeln und Institutionen besonders viel Rücksicht. Im Resultat benutzt Washington seine militärische Macht zur Durchsetzung einer globalen Ordnung (Ikenberry 2002: 49).

Das wesentliche ideologische Fundament dieser Strategie ist der "Neue Wilsonianismus“. Er wird zum einen von den internationalistischen Liberalen vertreten, die eine Ausweitung des amerikanischen Einflusses und ein Bündnis der Demokratien der Welt anstreben und zum anderen von den unilateralistischen Neokonservativen, die der Ansicht sind, eine aggressive amerikanische Führungsrolle sei für die Welt das Beste. Dieser Geist unterliegt den Initiativen des US-amerikanischen Kongresses gegen vermeintliche Schurkenstaaten, aber auch gegen zweifelhafte Verbündete, Sanktionen oder Boykotte zu verhängen (Pfaff 2001: 221-222). So rechtfertigt der „Neue Wilsonianismus“ den Unilateralismus: mit den Worten von Senator Jesse Helms ausgedrückt, „,besitzen demokratische und im Geist der Freiheit handelnde Staaten, allen voran die USA, eine unbegrenzte und keiner externen Kontrolle unterliegende Autorität für militärische Interventionen" (ebd.: 220-221).

Neben dem "Neue Wilsonianismus" lassen sich noch weitere ideologische Grundlagen der Bush-Strategie benennen: So haben die USA die Reformierung der Welt zu ihrer Mission gemacht, wobei sie sich der puritanischen Intoleranz ihrer kulturellen Ursprünge bewusst sind. Um diese fromme Mission zu erfüllen, erachtet Washington die Bekämpfung des "Terrorismus" an jedem Ort der Welt für notwendig. Allerdings ist es sehr viel schwieriger zu definieren, wer ein Terrorist ist, als es zunächst scheint. Von einer moralischen Perspektive aus betrachtet, ist der Krieg der Bush-Regierung gegen den Terror völ- 
lig unbefriedigend und ihre Rhetorik, die von einer eindimensionalen Perspektive auf ein multidimensionales Thema ausgeht, ist sowohl verzerrend als auch vereinfachend (Byford 2002: 34-43).

Weitere Gefahren, die angeblich bekämpft werden müssen, kommen hinzu. Sie alle werden durch Ad hoc-Theorien begründet: Bürgerkriege, die allgemeine Bedrohung des Westens durch den Islam, der globale Terrorismus, ein wiederauflebender chinesischer oder russischer Imperialismus, die internationale Kriminalität, Drogenhandel. Tatsächlich basieren diese Gefahren vor allem auf Spekulationen. Es sind in erster Linie Rechtfertigungen für die Aufrechterhaltung der allgegenwärtigen amerikanischen Militärpräsenz in der Welt, die vor allem der Regierungsbürokratie und privaten Unternehmen nutzt - allesamt Überlebende des Kalten Krieges.

Der „Neue Wilsonianismus" als Grundlage der Bush-Strategie enthält die sentimentale, größenwahnsinnige und unhistorische Vision einer Weltdemokratie. Zudem vertritt er die blasphemische Überzeugung, dass die amerikanische Nation von Gott geschaffen wurde, „um den Nationen der Welt zu zeigen, wie sie sich auf den Pfaden der Freiheit zu bewegen hätten" (Pfaff 2001: 227). Diese Wilsonsche Sentimentalität hatte in den vergangenen 80 Jahren desaströse Konsequenzen (ebd.: 226). Davon abgesehen leistet sie der Einschränkung staatlicher Souveränität Vorschub: Regierungen, die aus amerikanischer Sicht unakzeptabel handeln, wird ihre Souveränität abgesprochen. Mit anderen Worten, Bushs neoimperiale Strategie versucht die Welt (zumindest die Dritte Welt) in eine Einflusssphäre der Vereinigten Staaten zu transformieren. Das ist mit "Lateinamerikanisierung" der Welt gemeint.

Es sollte nicht vergessen werden, dass Großmächte, vor allem die Vereinigten Staaten, innerhalb ihrer traditionellen Einflusssphären üblicherweise gegen die Normen staatlicher Souveränität verstoßen haben, wann immer ihr nationales Interesse dies erforderte. Aber heute ist die Bush-Regierung der Ansicht „auch in globalem Rahmen auf diese Weise vorgehen zu können, indem sie sich die Autorität zugesteht, über den Verfall souveräner Rechte zu entscheiden - und diese Entscheidung auch noch präventiv zu treffen" (Ikenberry 2002: 53).

Über die Stabilität eines unipolaren Systems herrscht keine Einigkeit. Einige Autoren (Kupchan 2002) sind der Meinung, dass eine unipolare Welt nicht von Dauer sein kann und dass es sehr wahrscheinlich wieder eine Wende hin zur Multipolarität geben werde. Die USA seien mit zwei Risiken konfrontiert: Zum einen dem Aufstieg Europas und zum anderen der abnehmenden Unterstützung der amerikanischen Öffentlichkeit für den Internationalismus. Darüber hinaus könnten die wieder erstarkten linken und progressiven Kräfte in Lateinamerika und in anderen westlichen Ländern den Rückzug der USA von der globalen Bühne beschleunigen. Aus dem gleichen Grund ist der Diplomat und Historiker George Kennan, der vor über 50 Jahren geschrieben hatte, dass alle Nationen dem amerikanischen Beispiel, unter Wahrung ihrer jeweiligen 
Besonderheiten, folgen würden, heute davon überzeugt, dass die Welt nicht von einem einzigen politischen Zentrum aus geführt werden könne, egal wie groß dessen militärische Macht sei. Für Amerikaner, so fügt er hinzu, sei es "undurchdacht, prahlerisch und wenig wünschenswert', uns selbst als Zentrum der politischen Aufklärung und als Lehrer eines großen Teils der restlichen Welt zu betrachten" (Pfaff 2001: 224).

Zudem könnte die aggressive und schlichte Bush-Doktrin des „Entweder mit uns oder mit den Terroristen“ für die USA sehr gefährlich werden. Der IrakKrieg hat gezeigt, dass auch traditionelle Alliierte wie Deutschland und Frankreich als Feinde angesehen werden, wenn ihre Regierungen nicht mit der AuBenpolitik Bushs übereinstimmen. Auf der ideologischen Ebene - das werden wir später noch diskutieren - zerstört die direkte Ausübung der amerikanischen Macht zudem die Werte, auf denen die amerikanische Hegemonie basiert (Mandelbaum 2002: 72).

Sicherlich kann Unilateralismus kurzfristig effektiv sein, er kann die USA aber auch isolieren. Mit anderen Worten: Die Vereinigten Staaten sollten den möglichen Groll in Erwägung ziehen, den ein aggressiver unilateraler Kurs unter seinen Verbündeten hervorrufen kann (Brooks/Wohlfort 2002: 27). Zudem sollte sich das Land bewusst sein, dass für eine „Hypermacht“ nichts gefährlicher ist als Unilateralismus (Hoffmann 2002: 113) - denn ihre Macht kann zu einer destabilisierenden Kraft werden. Unipolarität ist geradezu eine Einladung an Herausforderer und oppositionelle Kräfte. Die amerikanische Position und das herrschende System werden in der Zukunft daher sehr wahrscheinlich in Frage gestellt und herausgefordert werden, wenn auch der Charakter der Herausforderung heute noch nicht vorhersehbar ist. Sie könnte von ähnlich fortgeschrittenen Gesellschaften ausgehen, aber auch von der inneren Erosion der Vereinigten Staaten. Möglicherweise wird sich wieder ein Pluralismus verschiedener Mächte etablieren - ob die Vereinigten Staaten nun dagegen Widerstand leisten oder nicht, und diese Entwicklung kann konstruktiv oder gewaltsam verlaufen (Pfaff 2001: 291-231).

Es gibt aber auch Autoren, die eine stabile Unipolarität für durchaus möglich halten, da die Vereinigten Staaten keinen Gegner haben, der ihnen durch seine Machtposition gefährlich werden könnte. Zudem ist die Dominanz der USA der derzeitige Status Quo. Die wichtigsten Mächte sind mit Washington verbündet und ziehen daraus ihren Nutzen. Und es gibt auch keinen Präzendezfall dafür, dass sich untergeordnete Mächte zusammengeschlossen hätten, um den Hegemon - nachdem sich dieser erst einmal etabliert hat - zu stürzen. Nach Ansicht dieser Autoren sind die USA einfach zu mächtig, als dass eine Machtbalance erreicht werden könnte. Zudem wäre der verlässlichste Weg zu einer solchen Balance die Erhöhung der Verteidigungsausgaben, seit 1995 sind aber die Rüstungsausgaben der meisten Großmächte relativ zum Bruttoinlandsprodukt gesunken. Und letztlich sei es eine Tatsache, dass die Vereinig- 
ten Staaten die Unipolarität anstreben und über genügend Ressourcen verfügen, um diese auch aufrechtzuerhalten (Brooks/Wohlfort 2002: 23-31).

Das große Problem jedenfalls besteht darin, dass Amerikas neoimperiale Strategie das Gefüge der internationalen Gemeinschaft zu zerstören droht (Ikenberry 2002: 44-45). Allerdings könnte Washington noch immer die Verärgerung über die Unipolarität mildern, vor allem durch den Abbau von Handelsschranken. Diese Politik „könnte als der Samthandschuh dienen, unter dem die eiserne Faust - die Macht Amerikas - steckt" (Brooks/Wohlfort 2002: 33). Zur Zeit gibt es für eine solche Orientierung jedoch keine Anzeichen. Die Welt wird daher auch in der nächsten Zukunft die nackte Faust von G.W. Bushs megalomanischer Außenpolitik zu spüren bekommen.

\section{Hegemonie in den Internationalen Beziehungen: der Neo-Gramscianische Ansatz}

Im Unterschied zur traditionellen Analyse der Internationalen Beziehungen geht ein jüngerer Ansatz von den theoretischen Überlegungen Antonio Gramscis aus, in denen das Konzept der Hegemonie eine zentrale Stellung einnimmt. Gramsci wandte diese Kategorie in seinen Forschungen zum italienischen Staat an, um zu verstehen, auf welche Art und Weise die herrschende Klasse herrscht. Er stellte heraus, dass eine solche Klasse gleichzeitig führend und dominierend sein muss: Führend auf moralischem und intellektuellem Gebiet, dominierend aufgrund ihrer wirtschaftlichen und politischen Macht. Hegemonie ergibt sich als Summe beider Elemente (Buci-Glucksmann 1980). Dieses HegemonieKonzept wurde nun von einigen Autoren auf den Bereich der Internationalen Beziehungen angewendet. Im traditionellen Ansatz, so ihre Kritik, wurde internationale Hegemonie lediglich als Dominanz eines mächtigen Staates innerhalb des Systems internationaler Beziehungen verstanden, der so Macht über die anderen Staaten gewinnt. Dies bleibe jedoch eine unvollständige Auffassung, da sie soziale Kräfte auf territoriale Einheiten reduziert. Das globale System müsse aber als Gesamtheit verstanden werden und die darin agierenden sozialen Kräfte als nicht territorial beschränkt (Gill 1993: 41-42).

Diesem Ansatz entsprechend muss eine Untersuchung der Internationalen Beziehungen zunächst mit der Definition der zu analysierenden Einheit beginnen. Gemäß Gramscis Methode bilden das Ensemble der durch die sozialen Strukturen geprägten sozialen Beziehungen oder die historische Situation die grundlegenden Einheiten dieser Analyse (Gill 1993: 24). Die aktuelle Situation auf dem Gebiet der Internationalen Beziehungen ist durch die Vorherrschaft der USA gekennzeichnet. In Anlehnung an Gramsci hat Robert W. Cox Hegemonie definiert als ein "Gefüge von Werten und Ansichten über das Wesen der Herrschaftsordnung, das ein ganzes System von Staaten und nichtstaatlichen Einheiten durchdringt" (Gill 1993: 41-42). In einer hegemonialen Ord- 
nung sind diese Werte und Ansichten relativ stabil und unhinterfragt. Gleichzeitig basiert diese Struktur - Gramscis Hegemonie-Konzept zufolge - auf einer Machtstruktur, in der ein Staat dominierend sein kann. Für die Herstellung von Hegemonie ist eine solche Dominanz aber nicht ausreichend. Es ist außerdem notwendig, dass die Art und Weise wie die dominierenden sozialen Schichten der dominierenden Staaten Handeln und Denken von den dominierenden sozialen Schichten der anderen Staaten akzeptiert wird. In ähnlicher Weise bezieht Giovanni Arrighi das Konzept einer Welt-Hegemonie auf die Macht eines Staates, Regierungsfunktionen über ein System souveräner Staaten ausüben zu können, wobei diese Macht mit der Ausübung einer intellektuellen und moralischen Führungsrolle - entsprechend der Führung der herrschenden Klasse bei Gramsci - verknüpft ist (Arrighi 1993: 149). Der hegemoniale Staat führt also ,ein System von Staaten in die von ihm gewünschte Richtung (...) und wird dabei als Vertreter eines allgemeinen Interesses wahrgenommen" (ebd.: 150).

In seiner Anwendung des Hegemonie-Konzepts auf die globale Ordnung unterscheidet Robert W. Cox zwischen hegemonialen und nicht-hegemonialen Perioden. In der jüngeren Geschichte war der Zeitraum von 1845-1875 eine hegemoniale Periode, es gab eine Weltwirtschaft mit England als Zentrum. Der anschließende Zeitraum von 1875-1945 war nicht-hegemonial. Das destabilisierte Gleichgewicht der Mächte gipfelte in zwei Weltkriegen, der Freihandel wurde durch Protektionismus abgelöst und die Weltwirtschaft in Wirtschaftsblöcke aufgeteilt. Von 1945-1965 folgte dann wieder eine hegemoniale Phase mit den Vereinigten Staaten als Zentrum. Die amerikanische Hegemonie sah sich schließlich in den 70er Jahren ernsten Problemen ausgesetzt so dass die USA sich bemühen mussten, ihre Vorherrschaft wieder zu festigen (Cox 1993a: 60-61).

In ihren Anfängen ist eine globale Hegemonie die räumliche Expansion einer nationalen Hegemonie, wie sie durch die dominierende soziale Klasse etabliert wird. Danach werden die ökonomischen und sozialen Institutionen, die Kultur und Technologie dieser nationalen Ökonomie zu Archetypen für den Rest der Welt. Die etablierte globale Hegemonie drückt sich dann in universellen Normen, Institutionen und Mechanismen aus, die den allgemeinen Verhaltensregeln für Staaten und den grenzübergreifend agierenden Kräften der Zivilgesellschaft zugrunde liegen. Diese Regeln befördern dann ganz offensichtlich die herrschende Produktionsweise (ebd.: 61-62).

Schließlich gibt es die theoretische Möglichkeit einer post-hegemonialen Ära, das heißt einer Zeit der Koexistenz verschiedener Zivilisationen, die auf jeweils spezifischen Intersubjektivitäten und eigenen Wertesystemen gegründet sind und die zudem ihren eigenen Entwicklungspfad verfolgen (Cox 1993b: 265). Dies wäre natürlich eine wünschenswerte Zukunft, unglücklicherweise gibt es zur Zeit keinetlei Anzeichen für eine derartige Entwicklung. 


\section{Die Entwicklung der US-amerikanischen Hegemonie}

Für eine Erklärung von Aufstieg und Fall der USA ist es nötig, die internationale Situation zu umreißen, die die Grundlage für die Entwicklung der amerikanischen Hegemonie bildet. Der Niedergang dieser Hegemonie beruht auf internen und externen Faktoren. Wichtigster interner Faktor ist das selbstzerstörerische, die eigene Vormachtstellung untergrabende Verhalten der USA, die Beschädigung gerade der Werte, auf denen die amerikanische Hegemonie basiert. $\mathrm{Zu}$ den bedeutendsten externen Faktoren zählen vor allem die zunehmende Multipolarität der Weltwirtschaft und die Verschlechterung der Beziehungen zwischen den Vereinigten Staaten und Lateinamerika. Vor diesem Hintergrund ist Washingtons Strategie zur Wiederherstellung der US-amerikanischen Hegemonie zu sehen.

Wallerstein (2002: 1) zufolge begann der Aufstieg der Vereinigten Staaten zur globalen Hegemonialmacht im Gefolge der weltweiten Rezession von 1873. Es kam zur Konfrontation zwischen Deutschland und England/Frankreich, die Arrighi zufolge in der kompletten Zersetzung des Weltmarkts und der Auflösung aller seiner tragenden Prinzipien mündete. Weltkrieg und russische Revolution setzten dem Freihandelsimperialismus und dem bisherigen internationalen System ein Ende.

„Wie England im frühen 19. Jahrhundert, so wurden die Vereinigten Staaten das erste Mal zu einer hegemonialen Macht, indem sie das internationale System zur Wiederherstellung der Prinzipien, Normen und Regeln des mit dem Westfälischen Frieden eingerichteten Staatensystems führten. Anschließend gingen sie dazu über, das wiederhergestellte System zu regieren und zu erneuern" (Arrighi 1993: 180). Das Resultat war das System des freien Unternehmertums. Das eindrucksvollste Produkt der US-amerikanischen Hegemonie markierte gleichzeitig ihre äußerste Grenze, denn es bedeutete auch das Ende des Westfälischen Systems der Staaten und den Beginn des Zusammenbruchs des zwischenstaatlichen Systems als dem fundamentalen Ort auf den sich „Weltmacht" bezieht (ebd.: 183).

Der Niedergang der USA als Weltmacht in den 70er und 80er Jahren wurde von verschiedenen Autoren wahrgenommen; es existiert eine nahezu unüberschaubare Vielfalt an Studien zum Aufstieg und Fall von "Hegemonien“, „Weltmächten" und "Großmächten". Eine der solidesten Erklärungen lieferte Immanuel Wallerstein ${ }^{2}$. Er konstatiert, dass die ökonomischen, politischen und militärischen Faktoren, die ursprünglich die Grundlage der amerikanischen Hegemonie darstellten, zwangsläufig zum Niedergang der Vereinigten Staaten führen müssen (Wallerstein 2002: 1). Das Verhalten der USA im Kampf gegen den „Terrorismus" habe diesen Niedergang lediglich beschleunigt.

2 Seit 1998 hat Wallerstein kürzere Kommentare zum globalen System verfasst (http:// fbc.binghamton.edu/commentr.htm), vgl. in Deutsch: Wallerstein (2003). 
Auf der anderen Seite haben Arrighi und Cox Studien kritisiert, in denen das Hegemonie-Konzept falsch angewendet wird. Arrighi bemängelt zum einen den Gebrauch des Begriffs „Hegemonie“ im Sinne von „Dominanz" und zum anderen die Unterstellung „einer grundsätzlichen Unveränderlichkeit desjenigen Systems, in dem die Macht eines Staates zu- und abnimmt" (Arrighi 1993: 148). Cox zufolge besteht der Fehler in der Debatte über die amerikanische Hegemonie in der fehlenden Unterscheidung zweier Bedeutungen des Begriffs: Die eine (konventionelle) Bedeutung sieht in Hegemonie die Dominanz eines Staates über andere Staaten. Die zweite an Gramsci orientierte Bedeutung beschreibt die Beschaffenheit eines Staatensystems, in dem der dominierende Staat seine Position aufrecht erhält, indem er an universalisierten Prinzipien festhält, die von einer ausreichenden Anzahl untergeordneter Staaten und sozialer Kräfte akzeptiert werden. Diese Bedeutung von Hegemonie impliziert eine intellektuelle und moralische Führungsrolle. So drückte sich die Hegemonie der USA in bestimmten Verhaltensgrundsätze aus, die weithin akzeptierbar wurden. Dennoch haben die zunehmend unilaterale US-Politik während der $80 \mathrm{er}$ Jahre und die deutlichen Interessenunterschiede zwischen den USA, Europa und Japan, sowie die offenere Unterordnung der Dritte-WeltLänder unter den ökonomischen und militärischen Druck des Westens die globalen Beziehungen verändert. „Die Macht der USA mag weder absolut noch relativ abgenommen haben, dennoch kann die Beschaffenheit des globalen Systems nicht länger als hegemonial im früheren Sinne beschrieben werden" (Cox 1993b: 264).

Unter Benutzung gramscianischer Konzepte analysierten Augelli und Murphy die Versuche der Reagan-Regierung, ihre Macht über die Dritte Welt zurückzugewinnen. Sie argumentieren, dass die dominierenden Klassen der USA in der zweiten Nachkriegsperiode einen soliden internationalen Block formiert haben. Dieser habe sich einen sicheren Platz in der Geschichte erobert: die „freie Welt", in deren Zentrum eine hegemoniale Allianz stand, die die herrschenden Klassen Westeuropas und Japans ebenso einschloss wie bestimmte Gruppen der Arbeiterklasse der OECD-Länder und die herrschenden Klassen vieler Länder der Dritten Welt (Augelli/Murphy 1993: 133).

Zu Beginn der 90er Jahre erreichte die US-amerikanische Hegemonie allerdings einen Tiefpunkt. Das frühere System hegemonialer Führung löste sich auf (Cox 1993b: 264). Im neu entstehenden System waren die USA eine „einsame Supermacht, der es an wahrer Macht mangelt, ein Führer der Welt, dem niemand folgt und den nur wenige respektieren und eine Nation, die gefährlich inmitten eines globalen und unkontrollierbaren Chaos treibt" (Wallerstein 2002: 3). Unter diesen Umständen, argumentiert Wallerstein, bleibt das Militär der stärkste und einzigste Trumpf in den Händen Washingtons. Der allmähliche Verlust der US-amerikanischen Rolle als ausschlaggebende Kraft in den globalen Angelegenheiten wird sich in den nächsten Jahren sicherlich weiter 
fortsetzen. Die Frage sei nicht, ob die amerikanische Hegemonie verschwinden wird, sondern ob die ÜSA diesen Abstieg werden hinnehmen können - ohne dabei der Welt und sich selbst Schaden zuzufügen (ebd.: 6-7).

Die beschriebenen Tendenzen müssen vor dem Hintergrund der Entwicklung des internationalen Systems gesehen werden, die gegenwärtig durch die Bewegung hin zu einer liberaleren und integrierteren globalen politischen Ökonomie charakterisiert ist. In diesem Zusammenhang tendieren die Hobbesschen und auch viele andere Staaten zu einer marktorientierten. Entwicklung sowie zur Internationalisierung einer selbstregulierten Zivilgesellschaft Lockescher Prägung (Gill 1993: 40).

Es muss auch berücksichtigt werden, dass die Weltordnung im 20. Jahrhundert durch eine ganze Serie tiefgreifender Identitätskrisen sowie durch nationale und internationale sozioökonomische Restrukturierungen sowohl in den entwickelten kapitalistischen Ländern als auch in den kommunistischen bzw. postkommunistischen Staaten und der Dritten Welt beeinflusst wurde. Der entscheidende Punkt ist dabei, dass alle diese Krisen durch die in der globalen Ökonomie agierenden Kräfte verknüpft sind (ebd.: 41).

Konzentrieren wir unsere Aufmerksamkeit nun auf die internen und externen Faktoren des Niedergangs der US-amerikanischen Hegemonie. Unter den ersteren muss vor allem der durch die USA selbst verursachte Schaden an den drei fundamentalen Werten herausgestellt werden, auf denen die amerikanische Hegemonie während des 20. Jahrhunderts basierte - Frieden, Demokratie und freie Märkte. Die gegenwärtige Welt wird durch diese drei großen Ideen dominiert: Frieden gilt dabei als beste Grundlage für die Beziehungen zwischen Ländern, Demokratie als der beste Weg, das politische Leben in den einzelnen Ländern zu organisieren und freie Märkte als unentbehrlich für die Produktion von Wohlstand (Mandelbaum 2002: 62). In diesen Punkten drückt sich die Universalität der amerikanischen Hegemonie aus - wenngleich die USA durch ihr Verhalten die gerade genannten Werte beschädigen und so ihre Vorherrschaft selbst untergraben.

Nehmen wir den Wert „Frieden“: in der ersten Hälfte des 20. Jahrhunderts haben die USA erfolgreich ihre Vorherrschaft in der Karibik und in Zentralamerika etabliert und dazu weit mehr als 50 bewaffnete Interventionen unternommen (Prevost/Oliva 2002: IX-X). Berücksichtigen wir auch das 19. Jahrhundert, dann verlängert sich die Liste der Opfer noch: Puerto Rico, Mexiko, Nicaragua, Panamá, Haiti, Kolumbien, Kuba, Honduras, die Dominikanische Republik, die Jungfraueninseln, El Salvador, Guatemala und Granada. Zoltan Grossman präsentiert auf seiner Homepage eine unvollständige Liste USamerikanischer Interventionen zwischen 1890 bis 1999; sie umfasst 132 Aktionen auf der ganzen Welt (www.zmag.org/CrisesCurEvts/interventions.htm). William Blum präsentiert in seinem Buch Rogue Nation eine Liste von $66 \mathrm{Mi}$ litärinterventionen zwischen 1898 bis 1991, in der folgende vier Arten bewaff- 
neter Intervention bereits herausgenommen wurden: a) Interventionen, bei denen die US-Truppen nicht die dominierende Kraft waren, b) die Bedrohungen mit Atomwaffen, c) vom CIA unternommene kriegerische Aktionen und d) die Interventionen der USA in Länder während des 1. und 2. Weltkrieges (www.sumeria.net/politics/usa.html). Mit diesen Daten sollte ausreichend demonstriert sein, dass die amerikanischen Regierungen bisher alles andere als besonders friedliebend gewesen sind. Ganz im Gegenteil haben sie systematisch eines der Prinzipien des Westfälischen Systems verletzt und damit die amerikanische Hegemonie selbst untergraben.

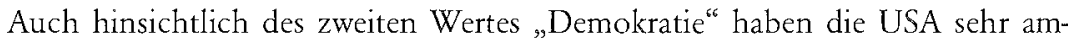
bivalente Positionen vertreten. Zwar hat sich der Einsatz für Demokratic im Laufe der Geschichte in der amerikanischen Politik einen festen Platz erobert. In der Zeit während und nach dem Kalten Krieg wurde der Begriff jedoch sehr eng gefasst und hinter andere nationale Interessen der USA zurückgestellt (Carothers 2003: 96). Der Einsatz für Demokratie war stets ein strategisches Instrument, um politische Ziele im Interesse der USA zu verfolgen. Damit geht aber auch die Legitimität des Konzepts verloren. In Bezug auf den Demokratisierungsprozess in Lateinamerika beispielsweise sollte nicht übersehen werden, dass dort die bedeutendste Demokratisierung in den frühen 80er Jahren stattfand, als Reagan noch immer Diktatoren zu unterstützen versuchte, die Jimmy Carter aus Menschenrechtsgründen aufgegeben hatte.

Aktuell ist es der Krieg gegen den Terror, der den US-amerikanischen Einsatz für Demokratie widersprüchlich erscheinen lässt. So zeigen die USA in bestimmten Ländern nur noch wenig Interesse an Demokratic, im Mittleren Osten dagegen ist dieses Interesse deutlich gewachsen. Sicher ist G.W. Bush nicht der erste US-Präsident mit einer gespaltenen Persönlichkeit hinsichtlich der Liebe zur Demokratie. Zweifellos aber spielt seine Persönlichkeit im Krieg gegen den Terror eine Rolle, die gefährlicher ist, als der Terrorismus selbst (ebd.: 96). Ein fundamentaler Aspekt von Demokratie innerhalb der internationalen Beziehungen betrifft die staatliche Souveränität. Eine demokratische internationale Ordnung muss von Staaten gebildet werden, die vor dem internationalen Recht gleich sind. Dabei kann dieses Recht nur existieren, wenn es von souveränen Staaten implementiert und befolgt wird. Verglichen mit dem Freihandelsimperialismus haben die Institutionen der US-amerikanischen Hegemonie Rechte und Macht der souveränen Staaten zu großen Teilen ausgehöhlt und sie stattdessen einer Vielzahl strenger Prinzipien, Normen und Regeln unterworfen, die von immer neuen supranationalen Organisationen diktiert werden. Dabei sind diese Organisationen inzwischen oft mächtig genug, um das zwischenstaatliche System nicht anerkennen zu müssen (Arrighi 1993: 182).

Kommen wir zum dritten Wert - der freien Marktwirtschaft. In dieser Disziplin sind die Vereinigten Staaten angeblich Weltmeister. Tatsächlich hat Washington in Vergangenheit und Gegenwart jedoch immer wieder Maßnahmen 
ergriffen, die freie Handelsbeziehungen ernsthaft einschränken oder sogar unterdrücken. Diese Tatsache wurde gleichermaßen von Mainstream-Ökonomen als auch von Autoren herausgestellt, die nicht die herrschende Lehre vertreten. Jagdish N. Bhagwati zufolge, einem Fürsprecher der weltweiten Handelsliberalisierung, hat Clinton nicht genug unternommen, um den freien Handel voranzutreiben. Regionale Handelsabkommen und Freihandelszonen bedeuteten nicht notwendigerweise auch freien Handel. Solche Abkommen können durchaus ,diskriminierend" sein (Bhagwati 2001).

Auf der anderen Seite diskutieren Altvater und Mahnkopf traditionelle Formen von Protektionismus, der zwischen den 40er und 80er Jahren in Lateinamerika durchaus üblich war und lenken gleichzeitig die Aufmerksamkeit auf den - keineswegs neuen - imperialistischen Protektionismus. Protektionistische Strategien wurden bereits in den 30er Jahren nach dem Zusammenbruch des Weltmarktes angewandt. Heute betrifft deren geläufigste Form den Bereich der nationalen Währungen. Gleichzeitig befördert die Herausbildung von Wirtschaftsblöcken in Europa, in Nordamerika und Lateinamerika, Asien und Afrika eine Fragmentierung des Weltmarktes (Altvater/Mahnkopf 1996: 387394 u. 409-502). In diesem Zusammenhang verhalten sich die USA ähnlich wie viele andere Großmächte, wenn sie auf die Konsolidierung des eigenen Wirtschaftsblocks hinwirken. Sie tun dies vor allem durch strenge Restriktionen in Bezug auf freie Märkte und freien Handel, auch innerhalb des Amerikanischen Blockes. Zu nennen wären bspw. die Kontrolle der Migration, die den Arbeitskräften aus Mexiko und anderen lateinamerikanischen Ländern aufgezwungen werden. Ein anderes Beispiel für einen direkten Eingriff in den Markt, der das freie Agieren der Wirtschaftssubjekte einschränkt, ist der Schutz der US-amerikanischen Landwirtschaft. Es kann daher niemand ernsthaft glauben, dass die USA die Freiheit der Wirtschaft respektieren. Freie Märkte und freier Handel sind auch nicht mehr als instrumentelle Werte im Dienste der ökonomischen und politischen Interessen der USA.

Unter den externen Faktoren, die die Hegemonie der USA untergraben, ist wahrscheinlich die multipolare Entwicklung der Weltwirtschaft der bemerkenswerteste. Seit den 80er Jahren beobachten wir die Herausbildung zweier großräumiger Wirtschaftssphären - eine in Europa und die andere in Ostasien - als Gegengewichte zur Dominanz der US-Wirtschaft und der nordamerikanischen Wirtschaftssphäre (Cox 1993b: 262). „Trotz Amerikas gewaltiger Wirtschaftsleistung in den 90er Jahren ist nun die Europäische Union das größte zusammenhängende Wirtschaftsgebiet auf der Welt und ihre Führungsrolle wird mit der Ausdehnung auf neue Mitglieder in den nächsten Jahren weiter anwachsen" (Bergsten 2001: 20-21). Das wirtschaftliche Gewicht von Ostasien ist vergleichbar mit dem der Vereinigten Staaten und der Europäischen Union. Das Ende des Kalten Krieges hat in diesem Zusammenhang zur Zuspitzung des ökonomischen Konfliktes beigetragen, denn mit dem Verschwinden der 
sowjetischen Bedrohung verschwand auch die Bedeutung des amerikanischen Schutzschildes über Europa und Asien. In der Konsequenz verschwand damit „das gemeinsame Interesse an Blocksicherheit, das traditionellerweise die Alliierten nach dem Krieg dazu anhielt, auch ihre wirtschaftlichen Differenzen zu überwinden" (ebd.: 21).

So zeigen die gegenwärtigen internationalen Verhältnisse alle Merkmale einer nicht-hegemonialen Periode. Das weltweite Handelssystem entwickelt sich nicht weiter, es findet eher eine Rückentwicklung statt. Nirgendwo gibt es ernsthafte multilaterale Verhandlungen auf diesem Gebiet. Der Rückschritt nimmt die Form eines intensivierten und diskriminierenden Regionalismus, zunehmender Handelsstreitigkeiten und Protektionismus an. Vor diesem Hintergrund haben auch in der internationalen Wirtschaft die Feindseligkeiten gegenüber den Vereinigten Staaten zugenommen (ebd. 23).

Ein zweiter erwähnenswerter externer Faktor ist die Verschlechterung der Beziehungen zwischen den USA und Lateinamerika. Zwar wurden diese seit Beginn der Regierung von Bush senior und dem Ende des Kalten Krieges freundlicher und kooperativer als je zuvor. Dennoch, so konstatiert Peter Hakim, gibt es unter der trügerisch glatten Oberfläche eine Reihe ernster Probleme. Es gibt genügend Gründe zur Annahme, dass der gute Wille und die Kooperation, die weite Teile der vergangenen zehn Jahre charakterisierten, gegenwärtig dahinschwinden (Hakim 2001: 48).

Ein wichtiger Grund waren die Finanzkrisen von 1995 und 1998. Das schrumpfende Wirtschaftswachstum und die rasant ansteigende Arbeitslosigkeit haben eine erfolgreiche Integration Lateinamerikas in die Weltwirtschaft großenteils verhindert (ebd.: 48). Dieses ökonomische Desaster war dabei eng verknüpft mit den weitläufig implementierten Reformprogrammen (bekannt als „Washingtoner Konsens"), die in vielen Ländern enttäuschende wirtschaftliche und soziale Konsequenzen hatten. „Für viele Lateinamerikaner bedeuten engere Beziehungen zu den USA schlicht eine Verschlechterung der bestehenden Situation. Der Widerstand gegen diese Reformen kann daher leicht in einen Widerstand gegen die Führung der USA umschlagen - und in Feindseligkeit gegenüber dem Internationalen Währungsfonds und der Weltbank, die beide als von den USA dominierte Institutionen angesehen werden“ (ebd.: 50). Nichtsdestotrotz ist man in fast jedem lateinamerikanischen Land der Ansicht, dass die ökonomische Zukunft vom Zugang zu US-amerikanischen Märkten und US-amerikanischen Investitionen abhängt.

Aber es bleiben ernsthafte Zweifel an der Verbindlichkeit Washingtons in der regionalen Zusammenarbeit. Die Beziehungen zwischen den Vereinigten Staaten und den meisten der lateinamerikanischen Länder, Mexiko ausgenommen, sind erheblich belastet. Viele Regierungen haben das Vertrauen in die USamerikanische Führung verloren und stellen die Verlässlichkeit Washingtons als Partner in Frage. Wenn jedoch die lateinamerikanischen Nationen ihre ö- 
konomischen und politischen Probleme nicht lösen können, werden sie aüch für Washington keine attraktiven Partner sein (ebd.: 60).

Unter den oben genannten Bedingungen versuchten die USA in den 80er Jahren ihre Hegemonie wiederherzustellen. Dies gelang zunächst durch eine effektive Anwendung ihrer wirtschaftlichen Macht, schließlich durch die Wiederbelebung der früheren, auf gemeinsamen Werten beruhenden Bezichungen zwischen den herrschenden US-amerikanischen Klassen und den herrschenden Klassen in Westeuropa und Japan. Das Ergebnis war jedoch, dass die amerikanische Vorherrschaft heute vor allem auf einer Dominanz beruht, die auf Gewalt und Ausbeutung basiert (Augelli/Murphy 1993: 133).

Der Niedergang der Dritten Welt durch monetaristische Politik, geringere Exporterträge und hohe Zinssätze, die in eine katastrophale Liquiditätskrise führten, war ein wesentlicher Bestandteil der US-amerikanischen Strategie. Daher hatten die USA nach der Schuldenkrise von 1982 für die Wiederherstellung ihrer Hegemonie innerhalb der westlichen Welt einen weitaus größeren Handlungsspielraum (ebd: 134). Mit dem Golfkrieg ging Bush senior schließlich noch einen Schritt weiter als Reagan, indem er ihn auch als Mittel der Konsolidierung der westlichen Allianz nutzte und der Minimierung der Gefahr, dass irgendein Dritte-Welt-Land sein Öl als Waffe nutzen könnte. Die BushRegierung versuchte dabei die herrschenden Klassen der weniger entwickelten Länder für den Westen zu gewinnen. Gleichzeitig vergab sie neue und erweiterte Rollen an zwischenstaatliche Organisationen, die mit der Bildung einer „neuen Weltordnung“ befasst waren (ebd.: 135-136).

Die Reintegration der herrschenden Klassen der Dritten Welt in den Westen kam vor allem durch die Unterstützung eines Elitenwechsels in den jeweiligen Ländern zustande. Eine eher leichte Aufgabe, da u.a. die lateinamerikanischen Eliten ihre Legitimation innerhalb ihrer Staaten verloren hatten. Gleichzeitig war die Hilfe von außen das Werkzeug für den Westen, mit dem diesen Ökonomien der Neoliberalismus aufgezwungen werden konnte. Die Idee der FTAA, wie Clinton sie in Miami erstmals vorstellte, sollte innerhalb dieser Strategie eine zentrale Rolle spielen, obgleich es auch kleine Fortschritte hin zu einem liberalisierten Nord-Süd-Handel gab (Bergsten 2001: 23).

Seit dem Regierungsantritt von George Bush junior mündeten diese USamerikanischen Versuche einer Rekonstruktion ihrer hegemonialen Position in einem verstärkten Unilateralismus, wie er zu Beginn dieses Artikels charakterisiert wurde. Dass sich damit jedoch die skizzierten Dilemmata der USHegemonie, ihre Untergrabung durch interne und externe Faktoren, auflösen lassen, ist nicht sehr plausibel, eher werden sich diese Dilemmata noch weiter verstärken. 


\section{Literatur}

Altvater, Elmar und Birgit Mahnkopf (1996): Grenzen der Globalisierung. Ökonomie, Ökologie und Politik in der Weltgesellschaft. Münster: Verlag Westfälisches Damptboot.

Arrighi, Giovanni (1993): The three hegemonies of historical capitalism, in Gill (1993a.), 148-185.

Augelli, Enrico und Craig N. Murphy (1993): Gramsci and international relations; a general perspective with examples from recent US policy toward the Third World, in: Gill (1993a), 127-147.

Bergsten, C. Fred (2001): America's Two-Front Economic Conflict, Foreign Affairs, 80: 2, 16-27.

Bhagwati, Jagdish (2001): The Wind of the Hundred Days: How Washington Mismanaged Glob. alization. Cambridge, Mass.: MIT Press.

Bohman, James (1997): The Public Spheres of the World Citizen, in James Bohman, Matthias Lutz-Bachmannr (eds.), Perpetual Peace: Essays on Kant's Cosmopolitan Ideal. Cambridge, Mass.: MIT Press.

Brooks, Stephen; William C. Wohlforth (2002): American Primacy in Perspective, Foreign Affairs, 81: 4, 20-33.

Buci-Glucksmann, Christine (1980): Gransci and the state. London : Lawrence and Wishart.

Byford, Grenville (2002): The Wrong War, Foreign Affairs, 81: 4, 34-43.

Carothers, Thomas (2003): Promoting Democracy and Fighting Terror, Foreign Affairs, 82: 1, 8497.

Cox, Robert W. (1993a): Gramsci, hegemony and international relations: an essay in method, in: Gill (1993a), 49-66.

Cox, Robert W. (1993b): Structural issues of global governance: implications for Europe, in: Gill (1993a), 259-289.

Gill, Stephen (1993): Epistemology, ontology, and the 'Italian school', in: Gill (1993a.), 21-48.

Gill, Stephen (ed.) (1993a): Gramsci, Historical Materialism and International Relations. Cambridge: Cambridge University Press.

Hakim, Peter (2001): The Uneasy Americas, Foreign Affairs, 80: 2, 46-61.

Hoffmann, Stanley (2002): Clash of Globalizations, Foreign Affairs, 81: 4, 104-115.

Ikenberry, John G. (2002): America's Imperial Ambition, Foreign Affairs, 81: 5, 44-60.

Kupchan, Charles (2002): The End of the American Exa: U.S. Foreign Policy After the Cold War, New York: Alfred Knopf.

Mandelbaum, Michael (2002): The Inadequacy of American Power, Foreign Affairs, 81: 5, 61-73.

Pfaff, William (2001): The Question of Hegemony, Foreign Affairs, 80: 1, 221-232.

Prevost, Gary und Carlos Oliva Campos (eds.) (2002): Neoliberalism and Neopanamaricanism. The View from Latin America, New York: Palgrave McMillan.

Wallerstein, Immanuel (2002): The Eagle Has Crash Landed, Foreign Policy, http:/ www.foreignpolicy.com/issue_julyaug_2002/wallerstein.html.

Wallerstein, Immanuel (2003): Auftakt zur globalen Anarchie, PROKLA 133, 33. Jg., Nr. 4, 565-574. 